\title{
Role of the interface region on the optoelectronic properties of silicon nanocrystals embedded in $\mathrm{SiO}_{2}$
}

\author{
N. Daldosso, ${ }^{1}$ M. Luppi, ${ }^{2, *}$ S. Ossicini, ${ }^{3}$ E. Degoli, ${ }^{3}$ R. Magri, ${ }^{2}$ G. Dalba, ${ }^{1}$ P. Fornasini, ${ }^{1}$ R. Grisenti, ${ }^{1}$ F. Rocca, ${ }^{4}$ L. Pavesi, ${ }^{1}$ \\ S. Boninelli, ${ }^{5}$ F. Priolo, ${ }^{5}$ C. Spinella, ${ }^{6}$ and F. Iacona ${ }^{6}$ \\ ${ }^{1}$ INFM and Dipartimento di Fisica, Università di Trento, I-38050 Povo (Trento), Italy \\ ${ }^{2}$ INFM-S ${ }^{3}$ and Dipartimento di Fisica, Università di Modena e Reggio Emilia, I-41100 Modena, Italy \\ ${ }^{3} I N F M-S^{3}$ and Dipartimento di Scienze e Metodi dell'Ingegneria, Università di Modena e Reggio Emilia, I-42100 Reggio Emilia, Italy \\ ${ }^{4}$ CNR-IFN, Istituto di Fotonica e Nanotecnologie del Consiglio Nazionale delle Ricerche, \\ Sezione "CeFSA" di Trento, I-38050 Povo (Trento), Italy \\ ${ }^{5}$ MATIS-INFM and Dipartimento di Fisica e Astronomia, Università di Catania, I-95123 Catania, Italy \\ ${ }^{6}$ CNR-IMM, Sezione di Catania, I-95121 Catania, Italy \\ (Received 30 May 2003; published 29 August 2003)
}

\begin{abstract}
Light-emitting silicon nanocrystals embedded in $\mathrm{SiO}_{2}$ have been investigated by x-ray absorption measurements in total electron and photoluminescence yields, by energy filtered transmission electron microscopy and by $a b$ initio total energy calculations. Both experimental and theoretical results show that the interface between the silicon nanocrystals and the surrounding $\mathrm{SiO}_{2}$ is not sharp: an intermediate region of amorphous nature and variable composition links the crystalline $\mathrm{Si}$ with the amorphous stoichiometric $\mathrm{SiO}_{2}$. This region plays an active role in the light-emission process.
\end{abstract}

DOI: 10.1103/PhysRevB.68.085327

PACS number(s): 73.22.-f, 61.10.Ht, 78.55.Mb, 78.67.Bf

\section{INTRODUCTION}

The silicon/silicon dioxide interface is one of the most studied interfaces because of the technological importance of thin silicon oxide films in semiconductor devices. Gate dielectrics, mask layers, sacrificial layers, insulators, cladding layers in wave guides, etc., are some of the various devices where the properties of the interface between $\mathrm{Si} / \mathrm{SiO}_{2}$ are exploited. Many different investigations have shown that, when a flat interface between $\mathrm{Si}$ and $\mathrm{SiO}_{2}$ is formed, this is extremely sharp (at single atom level) and extremely stable with respect to external agents. For this reason, silicon nanocrystals $\left(\mathrm{Si}-\mathrm{nc}\right.$ ) embedded in a $\mathrm{SiO}_{2}$ matrix have been used to form light emitting systems with superior properties with respect to porous silicon $(p-\mathrm{Si})$. Indeed $p$-Si is formed by $\mathrm{Si}$ nanostructures with a time dependent surface passivation. The quality and stability of the $\mathrm{Si} / \mathrm{SiO}_{2}$ interface in $\mathrm{Si}$-nc embedded in $\mathrm{SiO}_{2}$ matrix is what renders this system superior. The structural characterization of Si-nc and of their local environment is fundamental to understand the light emission mechanism in these systems.

The role of the interface on the electronic and optical properties of $\mathrm{Si}$-nc has been recently addressed in various experimental and theoretical studies. ${ }^{1-12}$ Many different findings have been reported. Wolkin et al. ${ }^{4}$ observed that oxidation introduces defect levels in the Si-nc band-gap which pin their emission energy. According to their model the defect levels are due to the formation of a Si-O double bond. The same conclusion has been reached in Refs. 9,12, whereas Vasiliev et al. ${ }^{10}$ have pointed out that similar results can be obtained also for $\mathrm{O}$ connecting two $\mathrm{Si}$ atoms (single bond) at the Si-nc surface. In Ref. 6, the assistance of Si-O vibrations at the interface has been proposed as the dominant path for recombination. Moreover interface radiative states have been suggested to play a key role in the optical gain of Si-nc. ${ }^{13,14}$
Here we present an experimental-theoretical study of light emitting $\mathrm{Si}$-nc embedded in $\mathrm{SiO}_{2}$. We provide evidence that the $\mathrm{Si} / \mathrm{SiO}_{2}$ interface is not sharp but a transition region composed of amorphous $\mathrm{Si}$ and a strained $\mathrm{SiO}_{2}$ coats the $\mathrm{Si}-\mathrm{nc}$ when they are embedded in $\mathrm{SiO}_{2}$. The experimental investigations show that this transition region participates to the light emission process; $a b$ initio density functional calculations indicate that the strained $\mathrm{SiO}_{2}$ layer around the $\mathrm{Si}-\mathrm{nc}$ introduces optically active states.

The paper is composed by an experimental part (Sec. II), where x-ray absorption and energy filtered transmission electron microscopy (EFTEM) measurements are presented, and by a theoretical part (Sec. III), where first principle calculations of Si-nc embedded in $\mathrm{SiO}_{2}$ are reported. Experimental and theoretical results are discussed in Sec. IV. Section V is devoted to conclusions.

\section{EXPERIMENTAL STUDY}

\section{A. Sample preparation and characterization}

Si-nc were produced by plasma enhanced chemical vapor deposition (PECVD) of $200 \mathrm{~nm}$ thick substoichiometric $\mathrm{SiO}_{x}$ on a $\mathrm{Si}$ substrate followed by a high-temperature annealing at $T=1250{ }^{\circ} \mathrm{C}$. This method is based on the temperatureinduced phase separation between $\mathrm{Si}$ and $\mathrm{SiO}_{2}$ in $\mathrm{SiO}_{x}$ films. The excess silicon atoms contained in the films, due to the high temperature, diffuse inside the oxide matrix and form nanometric silicon aggregates. Since the excess silicon is uniformly distributed along the film depth, the formation of nanocrystals does not depend on the film depth, and it is very poorly influenced by the presence of the $\mathrm{air} / \mathrm{SiO}_{x}$ and $\mathrm{SiO}_{x}$ /substrate interfaces. Experimental evidences of the depth-homogeneity of the films are provided by Rutherford backscattering spectrometry (RBS) and transmission electron microscopy (TEM). ${ }^{15}$ RBS data demonstrate the presence of 
a flat $\mathrm{Si}$ profile in the as deposited $\mathrm{SiO}_{x}$ films. The $\mathrm{Si}$ profile is not appreciably influenced by the subsequent annealing processes. Cross sectional TEM measurements demonstrate that $\mathrm{Si}$-nc are uniformly dispersed along all the film depth, without a remarkable depletion or segregation phenomena. A set of $\mathrm{SiO}_{x}$ films with different total atomic content of $\mathrm{Si}$ (35, 37, 39, and $42 \%$ ) was investigated after annealing at $1250{ }^{\circ} \mathrm{C}$ for $1 \mathrm{~h}$ in ultrapure $\mathrm{N}_{2}$ atmosphere. At increasing $\mathrm{Si}$ content, TEM measurements showed an increase of the Si-nc mean radius (from less than 1.0 to $1.7 \mathrm{~nm}$ ) and photoluminescence (PL) measurements a redshift (from 790 to $910 \mathrm{~nm}$ ) of the emission band. ${ }^{15,16}$

\section{B. X-ray absorption measurements}

X-ray absorption near edge structures (XANES) at the $k$ edge of $\mathrm{Si}$ were measured at Super-ACO (LURE) on the SA32 beam line. Two detection modes were simultaneously used: total electron yield (TEY) and photo luminescence yield (PLY). Both detection techniques measure secondary processes that follow the primary $\mathrm{X}$-ray absorption.

The TEY detection method is the most commonly used in $\mathrm{X}$-ray absorption spectroscopy (XAS) to study thick samples, because it measures the electrons escaping from the face of the sample directly exposed to x-ray irradiation. In the present case, we have measured the compensating current necessary to maintain a neutral charge on the sample. The sampling depth of TEY-XAS is mainly limited by the mean free path of the emitted/excited electrons: it is primarily dependent on the x-ray energy and, secondarily, on details of the sample. The process is quite complicated, because each primary Auger photoelectron may decade through a multistep path, producing a wide set of low energy electrons able to escape from the front side of the sample. Experimental tests and theoretical calculations of TEY-XAS sampling depth have been done in the literature for some materials. ${ }^{17-19}$ For silicon oxide, a careful experimental test has been done by Kasray et al., ${ }^{20}$ who recorded TEYXANES spectra both at $L_{2,3}$ and $k$ edge of $\mathrm{Si}$ from $\mathrm{SiO}_{2}$ layers anodically grown on Si substrates with thickness varying from 1 up to $65 \mathrm{~nm}$. The maximum sampling depth for TEY was estimated to be $\sim 5 \mathrm{~nm}$ for the $\mathrm{Si}-L$ and $\sim 70 \mathrm{~nm}$ for the $k$-edge regions. We have recently performed a similar test by thermally growing $\mathrm{SiO}_{2}$ layers over a $c$-Si wafer: at least up to $100 \mathrm{~nm}$, the contribution of the Si substrate, even though very reduced, is still detectable and decreasing in intensity with the $\mathrm{SiO}_{2}$ layer thickness.

Hence, differently from $L$ edges, TEY measurements performed at the Si $k$ edge have to be considered as sensitive to both near-surface and bulk $\mathrm{Si}$ atoms within a maximum range of the order of $100 \mathrm{~nm}$. In particular, TEY measurements provide average information about the absorbing atoms both in luminescent and nonluminescent sites. On the other hand, by the PLY-XAS method one deals with the X-ray excited optical luminescence (XEOL), i.e., one detects the visible photons emitted by a radiative center (single atom or nanostructure) that is excited after each single x-ray absorption process. ${ }^{21}$ In most cases, the integrated intensity of the whole XEOL band is collected as a function of the $\mathrm{x}$-ray energy. Moreover, when the signal is sufficiently intense, partial PLY-XAS spectra may be extracted, by selecting limited emission ranges from the XEOL spectra. Our experimental equipment has been especially built to this purpose: it is based on a CCD detector mounted on a dispersion spectrometer. In this configuration, one luminescence spectrum is collected and stored at each X-ray energy point, and many partial PLY-XAS spectra can be obtained from the same measure by an off-line analysis. ${ }^{22-24}$

For the energy range of the $k$ edge of $\mathrm{Si}$, the sampling depth of the PLY-XAS is only determined by the absorption length of the $\mathrm{X}$-ray photons, which is $\sim 1.3 \mu \mathrm{m}$ after the absorption edge: ${ }^{19,25}$ thus the whole film thickness is taken into account.

The PLY-XAS detection technique is very useful when one may take advantage of its peculiar site selectivity. In well selected cases, in fact, only a subset of the absorbing atoms in the sample may contribute to the PLY signal: the atoms in the proximity of the luminescent center. The more useful application of PLY-XAS method is obtained by a detailed comparison with XAS spectra measured by a second detection technique. Some of the present authors have widely used TEY- and PLY-XAS to investigate porous silicon, ${ }^{22-27}$ where only a subset of the silicon atoms is luminescent. The origin of light emission in this case is due to quantum confinement, i.e., to the very reduced size of the nanostructures present in the porous layer. On the basis of the previous experimental evidences, we will interpret in this paper our obtained PLY and TEY-XANES spectra of Si-nc embedded in a silica matrix: as we will see, the system shows XAS spectra very different from $p$-Si; however, the luminescence is due to the same effect, quantum confinement, as for $p$-Si.

Results. In the Si-nc samples, two well separated $k$ absorption edges are present: the first, starting at $1839 \mathrm{eV}$, is due to the absorption by $\mathrm{Si}$ atoms bonded to $\mathrm{Si}$; the second is due to the absorption by $\mathrm{Si}$ atoms in $\mathrm{SiO}_{2}$ and is characterized by a white peak at about $1847 \mathrm{eV}$, followed by a flat region before the beginning of the EXAFS oscillations. The white peak is very sensitive to small changes in the structure or stoichiometry that induce changes in the electron density of states. A quantitative interpretation of these changes in $a-\mathrm{SiO}_{2}$ spectra is quite difficult, because the available $a b$ initio calculations of XANES are not accurate enough. Moreover, the knowledge of medium-range order in silica is still an open problem. Figure 1 shows the normalized TEY and PLY XANES spectra of one of Si-nc samples (37\% at Si), for a direct comparison with TEY reference spectra of silicon and $a-\mathrm{SiO}_{2}$.

The full set of results for all the $\mathrm{Si}-\mathrm{nc}$ samples is presented in Fig. 2. According to a well established procedure, the normalization among the various spectra has been done in the flat region centered at $1855 \mathrm{eV}$. The PLY-XAS signals have been obtained by integrating the whole intensity of the luminescence band at each X-ray energy step. Due to the very low intensity, the acquisition time was set to $30 \mathrm{~s}$. TEY and PLY measurements were done simultaneously, hence the relative positions of edge and XANES features are not affected by monochromator uncertainties or resolution effects. 


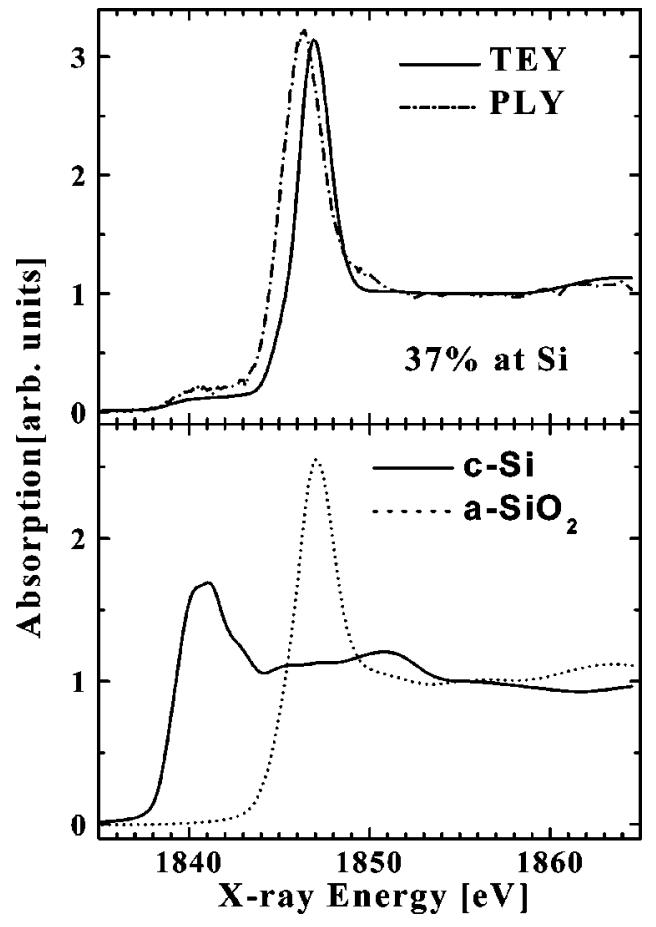

FIG. 1. Comparison of the $\mathrm{x}$-ray absorption spectra measured in TEY and PLY mode for the nc-Si sample with $37 \%$ at Si (top) with reference TEY spectra of $c-\mathrm{Si}$ and $a-\mathrm{SiO}_{2}$ (bottom). Experimental spectra have been normalized in the $(1850-1860 \mathrm{eV})$ region.

Let us discuss firstly the region of the low energy edge. We exclude any contribution to both TEY and PLY signals by the silicon substrate. In fact, the sufficiently large thickness of samples $(200 \mathrm{~nm})$ prevents the electron's escape from

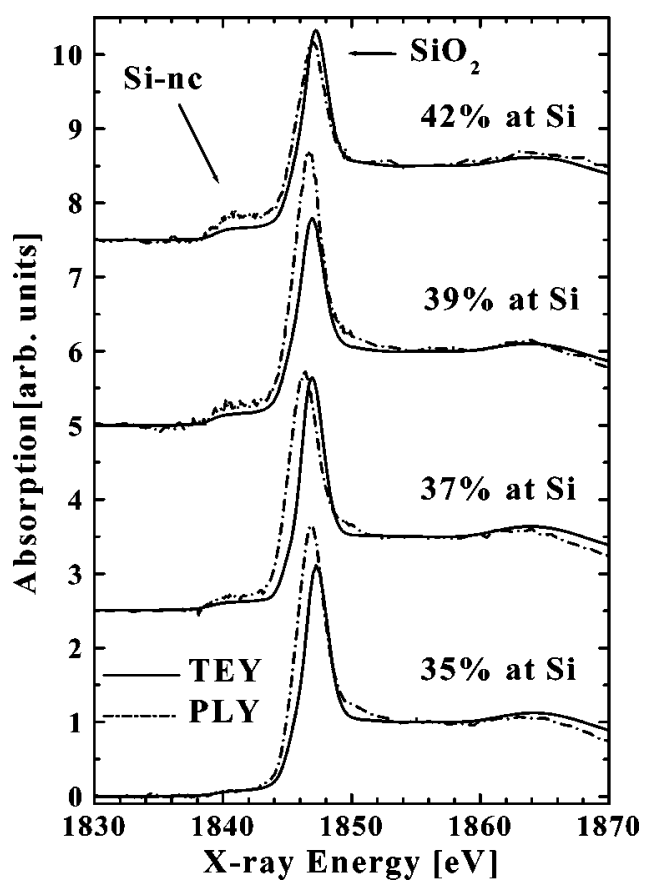

FIG. 2. X-ray absorption spectra for the various Si-nc samples measured in TEY and PLY mode, normalized in the (1850-1869 $\mathrm{eV})$ region.

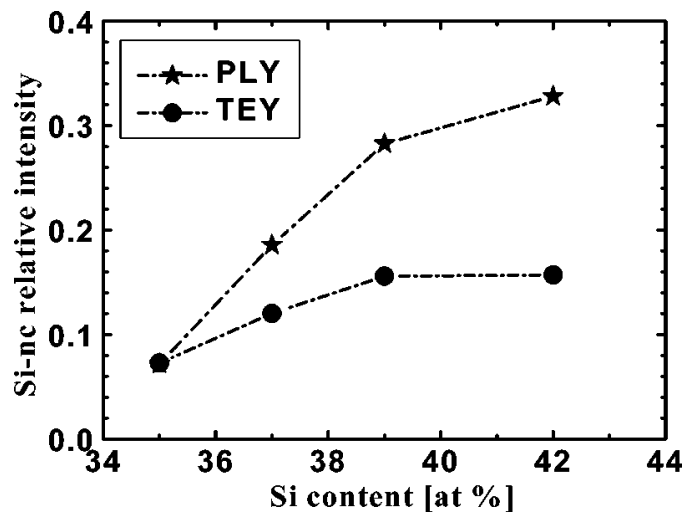

FIG. 3. Relative intensity at $1841 \mathrm{eV}$ of the contribution of Si atoms clustered in Si nanodots.

the substrate and the luminescence of the $c$-Si wafer is negligible. In this region, the relative intensity of the absorption coefficient depends on the fraction of $\mathrm{Si}$ atoms clustered in $\mathrm{Si}$-nc with respect to those coordinated to $\mathrm{O}$ in $\mathrm{SiO}_{2}$. This contribution grows with increasing $\mathrm{Si}$ content for both TEY and PLY spectra, as documented in Fig. 3. From TEYXANES and Rutherford backscattering spectrometry data, a quantitative analysis of the amount of $\mathrm{Si}$ atoms in the $\mathrm{Si}$-nc has been recently reported in Ref. 28. As well as this, we can compare here the TEY and PLY spectra. Figure 3 shows that the intensity of PLY signal is significantly higher than that of TEY; moreover, the difference grows with increasing the Si excess. This means that the concentration of $\mathrm{Si}$-nc in the luminescent part of the sample is larger than in the whole one.

Let us now look at the overall spectra. It is evident from Figs. 1 and 2 that the XEOL is a consequence of X-ray absorption in both $\mathrm{Si}$-nc and $\mathrm{SiO}_{2}$. Indeed the PLY-XANES signal shows a significant increase at both absorption edges. This is a noticeable difference with respect to porous silicon. In fact, for $p$-Si, even in aged or slightly oxidized samples, the PLY-XANES spectra obtained by monitoring the red XEOL band do not show a positive contribution at the $\mathrm{SiO}_{2}$ absorption edge ${ }^{19,30}$ only with anodically oxidized $p$-Si samples, we were able to detect a small positive contribution, ${ }^{22}$ while samples with a much thicker oxide layer showed a decreasing intensity ${ }^{19,22}$ and a typical reverse-edge behavior. ${ }^{21}$ The different behavior for Si-nc in $\mathrm{SiO}_{2}$ and $p$-Si indicates a different quality of the passivating oxide layer in the two materials.

Figure 2 points out another important characteristic of the Si-nc: TEY and PLY XANES spectra measured on the same sample contains significant differences in line shape and spectral position of the $\mathrm{SiO}_{2}$ related white peak at about 1847 $\mathrm{eV}$. We attribute these differences to the presence around the Si-nc of a modified $\mathrm{SiO}_{2}$ region participating to the light emission process, but not extending to the whole silica matrix: otherwise no difference in PLY and TEY spectra would have been observed. We note, in particular, that for all the studied samples the PLY XANES signal grows up at lower energy than TEY and that a remarkable difference in the relative intensity of the PLY and TEY peaks is evident for all 
the samples. The energy difference may be evaluated by monitoring the maximum of the first derivative: it is about $0.4-0.5 \mathrm{eV}$, with small variations from sample to sample.

The interpretation of these changes may be done on the basis of a phenomenological comparison with similar evidences published in the literature. It is worth noting that a low-energy shift of the absorption edge of $\mathrm{SiO}_{2}$ and a decrease in intensity of the white peak were clearly observed in densified silica. ${ }^{29}$ In that case, changes were mainly attributed to a reduction of the midrange $\mathrm{Si}-\mathrm{Si}$ interatomic distances. Following this interpretation, we may conclude that the differences between PLY and TEY XANES in the present samples indicate that the $\mathrm{SiO}_{2}$ absorbing sites able to excite the photoluminescence have a local structure different from the average one monitored by TEY. Furthermore, the differences between TEY and PLY in the region after the white peak (at about $1850 \mathrm{eV}$ ) confirm this conclusion, because they may be explained by a modification of medium-range structures. $^{31,32}$

Thus we interpret our data with a structural model where the silicon nanodots are coated by a modified $\mathrm{SiO}_{2}$ region, which plays an active role in the luminescence. The $\mathrm{Si} / \mathrm{SiO}_{2}$ interface is not sharp but a transition region should be considered where a strained $\mathrm{SiO}_{2}$ is formed.

In order to evaluate the thickness of the transition region, the ratio $A$ between the maximum of the $P L Y_{\mathrm{Si}-n c}$ absorption (measured at $1841 \mathrm{eV}$ ) and the absorption $P L Y_{\text {tot }}$ at $1855 \mathrm{eV}$ (which accounts for Si-nc and the $\mathrm{SiO}_{2}$ cap shell) was determined. $A$ can be also computed by considering a sphere of radius $R$ with a core of radius $r$ and assuming that the luminescence yield is the same for $\mathrm{Si}$-nc (core) and $\mathrm{SiO}_{2}$ (cap shell), and that the PLY signal is proportional to the atomic absorption coefficient $(\mu)$ and to the volume. Hence,

$$
A=\frac{\mathrm{PLY}_{\mathrm{Si}-\mathrm{nc}}}{\mathrm{PLY}_{\text {tot }}}=\frac{\mu_{\mathrm{Si}} V_{\text {core }}}{\mu_{\mathrm{Si}} V_{\text {core }}+\mu_{\mathrm{SiO}_{2}} V_{\text {shell }}} .
$$

By using McMaster tables, ${ }^{33}$ one obtains

$$
R=r \sqrt{[3}(177 / A)-0.74] .
$$

The ratio $A$ can be easily determined from PLY measurements; the core radius $r$ is that estimated from TEM measurements ${ }^{15,16}$ and hence an evaluation of the transition region size $(R-r)$ can be done. This turns out to be about 1 $\mathrm{nm}$ for all the samples.

\section{High resolution EFTEM analysis}

Very detailed information about the Si-nc structure can be obtained by collecting high resolution TEM images. In particular, the image shown in Fig. 4 has been obtained by using the energy filtered TEM (EFTEM) technique. This technique involves the use of a conventional TEM coupled with an electron energy loss spectrometer, and it allows one to create an image by using only electrons that have lost a specific amount of energy due to the interaction with the sample. As a consequence, a chemical mapping of all the species present in a sample with the very high spatial resolution typical of TEM can be obtained. EFTEM represents a particularly suit-

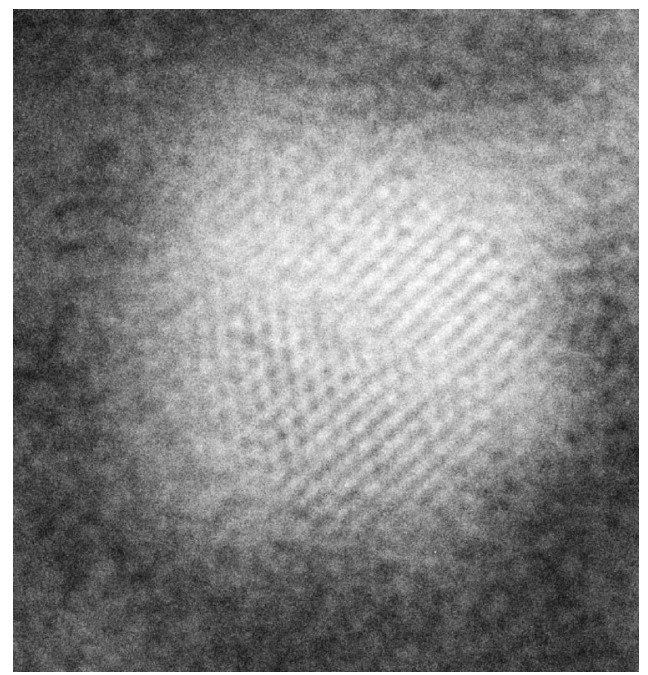

FIG. 4. High resolution energy filtered TEM image of a Si-nc after annealing at $1250{ }^{\circ} \mathrm{C}$. The presence of the $\mathrm{Si}$ (111) reticular planes is clearly visible in the nc core. The distance between two adjacent (111) planes is $0.32 \mathrm{~nm}$.

able method for the detection of Si-nc dispersed in a silica matrix. The electron energy loss spectrum coming from a sample consisting of $\mathrm{Si}$-nc embedded in $\mathrm{SiO}_{2}$ is dominated by plasmon loss peaks centered at about 17 and $23 \mathrm{eV}$. They correspond to the $\mathrm{Si}$ and $\mathrm{SiO}_{2}$ contributions, respectively. A Si map can be therefore obtained by using an energy window centered at the Si plasmon loss. A complex Si-nc structure appears from the analysis of the high resolution image obtained under energy filtered conditions shown in Fig. 4, referring to a Si-nc coming from a $\mathrm{SiO}_{x}$ sample after annealing at $1250{ }^{\circ} \mathrm{C}$. In particular, the presence of the $\mathrm{Si}$ (111) planes indicates the presence of a crystalline core. Furthermore, this core is surrounded by an external shell. This shell is about 1 $\mathrm{nm}$ thick and it is characterized by the absence of reticular planes. Under the employed experimental conditions, this experiment reveals that a sharp $\mathrm{Si}-\mathrm{nc} / \mathrm{SiO}_{2}$ interface does not exist, but that an intermediate region is present.

\section{THEORETICAL STUDY}

The calculation of the electronic and optical properties of $\mathrm{Si}$-nc embedded in a $\mathrm{SiO}_{2}$ matrix is a formidable task. In literature computations on $\mathrm{Si}-\mathrm{O}$ systems have concerned mainly with the $\mathrm{Si} / \mathrm{SiO}_{2}$ interface between $\mathrm{Si}$ and $\mathrm{SiO}_{2}$ layers or multilayers. ${ }^{8,34-36}$ Recent works have been addressed to $\mathrm{Si}$ clusters or nanocrystals covered by oxygen, ${ }^{4,9-12}$ but they deal with isolated systems. Here we have focused our attention on the role that interface can have on the properties of $\mathrm{Si}$ nanocrystals considering as surrounding environment a $\mathrm{SiO}_{2}$ matrix. To our knowledge only two works have studied, up to now, embedded nanostructures. In the first, ${ }^{37}$ a spherical square-well potential, to simulate different silicon quantum dots embedded in silicon dioxide, and effective mass approximation, to describe the two materials, have been used; thus the details about the atomic structure of the system are not investigated. In the second work ${ }^{38}$ an $a b$ initio DFT study on the electronic and optical properties of $\mathrm{Si}$ and Ge dots, 
with different sizes, inside a different $(\mathrm{SiC})$ host matrix is carried out. The ground state results are obtained by electronic energy minimization at fixed system geometry, i.e., without relaxing the ion positions. In the present work we do a step forward considering the relaxation of the complete system by total energy minimization. A simple model for Si-nc embedded in crystalline $\mathrm{SiO}_{2}$ is presented highlighting the relation between the structural and the electronic properties.

\section{A. The Model}

The first problem we had to overcome in modelling Si-nc embedded in $\mathrm{SiO}_{2}$ is the choice of the $\mathrm{SiO}_{2}$ host matrix type, the second is related to the type of $\mathrm{Si} / \mathrm{SiO}_{2}$ interface. A lack of symmetry with respect to a crystalline situation and the great amount of atoms together with large cell dimensions make the system intractable by $a b$ initio DFT calculations. For this reason we decided to consider a crystalline $\mathrm{SiO}_{2}$ in order to exploit the symmetry and to keep the supercell size as small as possible. The choice between the available crystalline forms was the answer to the second problem, the type of $\mathrm{Si} / \mathrm{SiO}_{2}$ interface. From a theoretical point of view the studies on the interface between $\mathrm{Si}$ surface and different types of crystalline $\mathrm{SiO}_{2}$ suggest the tendency of minimizing the number of defects at the interface..$^{34,36,39}$ To achieve this situation we decided to take as host material the $\mathrm{SiO}_{2} \beta$-cristobalite (BC) which is the form closer to the crystalline silicon structure. The peculiarity of the $\mathrm{BC}$ is in fact to have a diamondlike symmetry, i.e. tetrahedral coordination, which can ensure one of the simplest $\mathrm{Si} / \mathrm{SiO}_{2}$ interface. ${ }^{36,40}$ To model a Si-nc embedded in the $\mathrm{SiO}_{2}$ matrix, we designed a simple-cubic supercell of $\mathrm{BC}$ repeating twice its unit cell along each Cartesian axis. We obtained in this way a cell side of $14.32 \AA$. Then we cut out from the $\mathrm{SiO}_{2}$ structure $12 \mathrm{O}$ atoms and we "linked" together the Si atoms left with dangling bonds. The resulting supercell was thus made of $64 \mathrm{Si}$ and $116 \mathrm{O}$ atoms with $10 \mathrm{Si}$ "bonded together" to form a small Si-nc surrounded by a crystalline $\mathrm{SiO}_{2}$ matrix. The achieved $\mathrm{Si} / \mathrm{O}$ ratio was thus $35 \%$. The $\mathrm{Si}$-nc tetrahedral coordination was ensured by the peculiar symmetry of the $\mathrm{BC}$ but the $\mathrm{Si}-\mathrm{Si}$ bond length were highly strained compared to bulk-Si $(3.10 \AA,+33 \%)$. There was no defect at the interface and all the $\mathrm{O}$ atoms at the Si-nc surface were single bonded with the $\mathrm{Si}$ atoms. This initial geometry was then optimized performing total energy pseudopotential calculations.

\section{B. Computational methods}

Our DFT total energy calculations were based on the plane wave pseudopotential code CASTEP, ${ }^{41,42}$ using ultrasoft pseudopotentials suitable for high performance solid state calculations. ${ }^{42,43}$ The geometry optimization was performed using a GGA-PBE (Ref. 44) exchange and correlation (XC) treatment and a real space representation for the pseudopotentials. The kinetic energy cutoff was set at $260 \mathrm{eV}$ and we considered one special $k$ point only. No external pressure or stress were applied and we left all the atom positions and the cell dimensions free to move (keeping fixed the cell angles).
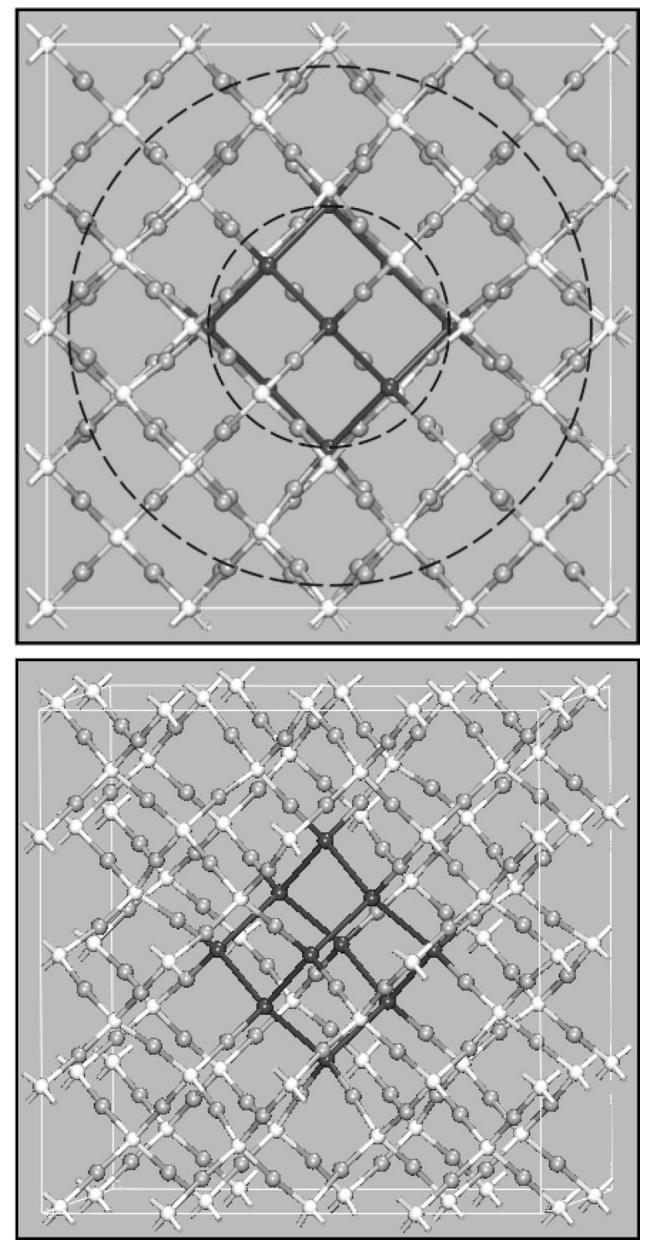

FIG. 5. Optimized structure for the $\mathrm{Si}-\mathrm{nc}$ in $\mathrm{SiO}_{2} \mathrm{BC}$ supercell: top view (top) and side view (bottom). White balls stand for $\mathrm{Si}$ atoms, gray for $\mathrm{O}$ and black for $\mathrm{Si}$ of $\mathrm{Si}-\mathrm{nc}$. The two dashed circles (top) delimitate the $\mathrm{Si}-\mathrm{nc}$ and the cap-shell region.

Once obtained the relaxed structure we determined the band structure considering 24 empty states, for a total of 500 bands, and 29 special $k$ points, using GGA-PBE XC treatment.

The optical properties were determined considering only direct interband transitions. We chose an LDA approach, considering 24 empty states, 20 special $k$ points, and using a reciprocal space representation for the pseudopotentials. No $\mathrm{XC}$ corrections or excitonic and local field effects were included. In order to understand the character of the states displayed by the band structure results and involved in the optical transitions we also calculated the spatial distribution of the Kohn-Sham orbitals square modulus, using the same prescription of the optical properties runs but with one special $k$ point.

\section{Results}

Structural Properties. The optimized supercell for the $\mathrm{Si}_{10} / \mathrm{SiO}_{2}$ is reported in Fig. 5. The initial crystalline structure of the $\mathrm{Si}_{10}$ is preserved after the total energy minimization procedure with a final Si-Si bond length of $2.67 \AA$, $14 \%$ strained with respect to bulk-Si. This huge contraction with 


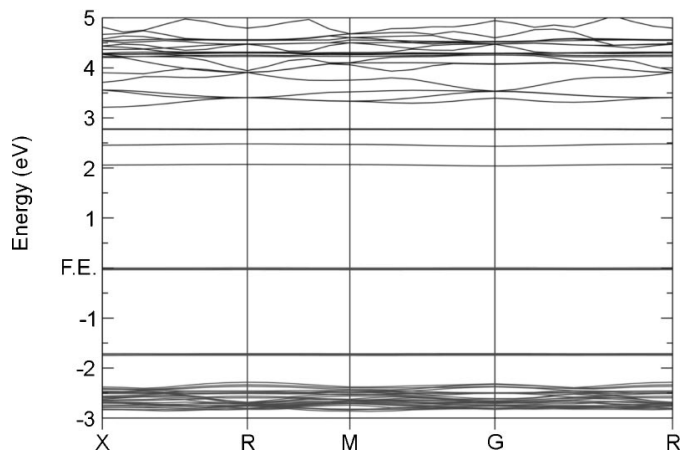

FIG. 6. Electronic band structure along high symmetry points of the $\mathrm{BZ}$ for $\mathrm{Si}-\mathrm{nc}$ in $\mathrm{SiO}_{2} \mathrm{BC}$ at relaxed geometry. F.E. indicates the Fermi energy of the system.

respect to the initial situation causes a complex deformation of the $\mathrm{SiO}_{2}$ matrix around the $\mathrm{Si}-\mathrm{nc}$, both in bond lengths and angles. The general trend, even if locally the situation can be different, is a contraction of the BC structure in the vicinity of the Si-nc, especially the second and third nearest neighbors distances (3\% compressed). This sort of densification is in agreement with the observations of Lagarde and co-workers, previously discussed. ${ }^{29}$ The effect of strain on the 1s core-level binding energy of $\mathrm{Si}$ in bulk $\mathrm{SiO}_{2} \mathrm{BC}$ has recently been calculated. ${ }^{16} \mathrm{~A}$ compression of about $2-3 \%$ of the equilibrium lattice constant results in a shift of the absorption edge of about $0.5 \mathrm{eV}$. Not all the $\mathrm{SiO}_{2}$ atomic positions are affected by the relaxation process. From the analysis of the final geometry clearly emerges that the initial symmetry of the $\mathrm{BC}$ is preserved in the outer regions of the cubic supercell. Both interatomic distances and bond angles result in fact to be practically unchanged after the minimization process. This, in principle, could be related to the constrains imposed by the supercell. These atoms are in fact mostly on the cell boundaries. Nevertheless the choice of leaving free to relax the cell dimensions during the optimization procedure makes us self confident that this result is not a consequence of the calculation technique.

Thus a transition region is present in the relaxed supercell. It is constituted by a distorted BC that surrounds the Si-nc. The thickness of the transition region (equal to the mean distance between the Si-nc and the outer crystalline BC atoms) is of the order of $8-9 \AA$. Figure 5 (top) highlights the final geometry achieved with the Si-nc core, the transition region of stressed $\mathrm{SiO}_{2} \mathrm{BC}$ and the outer crystalline $\mathrm{SiO}_{2}$ BC.

Electronic and optical properties. Figure 6 shows the electronic properties of the relaxed supercell. A clear semiconductor behavior, with a GGA-PBE energy gap of $2.07 \mathrm{eV}$ (1.96 eV in LDA), is observed, while in the starting lattice configuration (no relaxation), the high strain imposed on the $\mathrm{Si}$-nc induced a metallic behavior. The $E_{g}$ value for the relaxed system has to be compared with the calculated GGAPBE $E_{g}$ for the $\mathrm{SiO}_{2}$ BC bulk, $5.84 \mathrm{eV}$ (5.48 eV in LDA). The strong reduction is caused by the formation at the conduction (CB) and valence band (VB) edges of confined, flat, states related to the Si-nc presence. Deep inside each band, instead, the typical behavior of the $\mathrm{SiO}_{2}$ BC-bulk is still

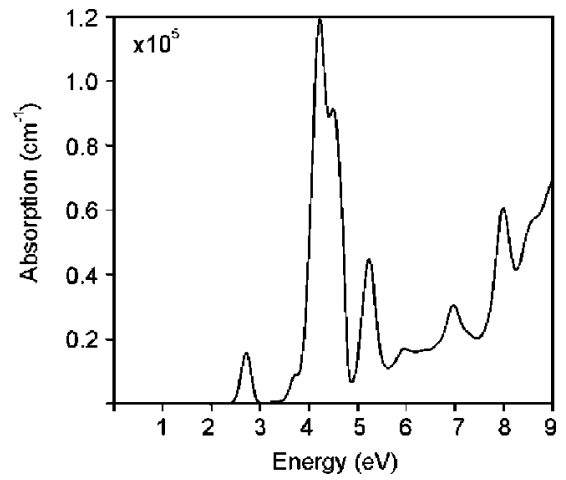

FIG. 7. Calculated absorption spectrum (direct interband transitions) for the Si-nc in $\mathrm{SiO}_{2} \mathrm{BC}$ at relaxed geometry. An artificial Gaussian broadening of $0.05 \mathrm{eV}$ has been applied.

recognizable (see, for example, the band plot at about $3 \mathrm{eV}$ below the Fermi energy). This shows how the Si-nc presence is able to strongly modify not only the structural but also the electronic properties of the matrix. We can estimate the valence band offset between the $\mathrm{Si}$-nc and the $\mathrm{SiO}_{2}$ matrix in $2.5 \mathrm{eV}$. The calculation of the ground state behavior for a Si-nc with the same final relaxed geometry of the $\mathrm{Si}_{10} / \mathrm{SiO}_{2}$ but passivated with hydrogen and surrounded by vacuum shows a completely different distribution of the energy levels and a bigger $E_{g}(4.03 \mathrm{eV}$ in LDA). This highlights how also the $\mathrm{SiO}_{2}$ matrix can influence the $\mathrm{Si}$-nc electronic properties, showing the strong interplay between the embedded system and the host mean.

In Fig. 7 we report the calculated absorption spectrum. A first very weak peak is observed around $2.1 \mathrm{eV}$ which corresponds to the transition involving the HOMO (highest occupied Kohn-Sham orbital) and LUMO (lowest unoccupied Kohn-Sham orbital) states. The first really pronounced feature is centered at about $2.7 \mathrm{eV}$ and is mainly due to transitions from the HOMO to the $\mathrm{LUMO}+3$ and $\mathrm{LUMO}+4$ states, where by LUMO +3 or LUMO +4 are meant the third or fourth unoccupied orbitals at energies higher than the LUMO. In order to have an idea of the character of these states, we have calculated the spatial distribution of the orbitals square modulus (Fig. 8). The HOMO state is mainly localized on the Si-nc region but some weight on the $\mathrm{O}$ atoms at the $\mathrm{Si}-\mathrm{nc} / \mathrm{SiO}_{2}$ interface is evident. The LUMO +3 (in particular) and the LUMO +4 show instead a totally different behavior. The distributions lose part of their Si-nc character and tend to be localized mostly on some oxygen atoms at the Si-nc surface, i.e., on the $\mathrm{SiO}_{2}$ cap shell. Thus it follows that the transition region around the Si-nc can participate in the optical activity of the system directly. Moreover, the orbital localization on the Si-nc region supports the view of nearband-edge states related to the Si-nc, while the contribution on the $\mathrm{O}$ atoms points out the role of the surrounding oxide shell on the Si-nc levels organization suggested by the band structure analysis.

\section{DISCUSSION}

Let us review and discuss the main findings of our study. (1) Thermal annealing of $\mathrm{SiO}_{x}$ films at $T=1250{ }^{\circ} \mathrm{C}$ 


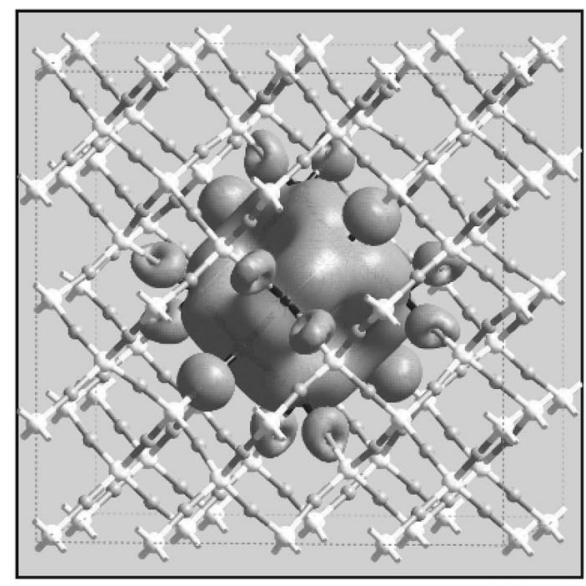

HOMO

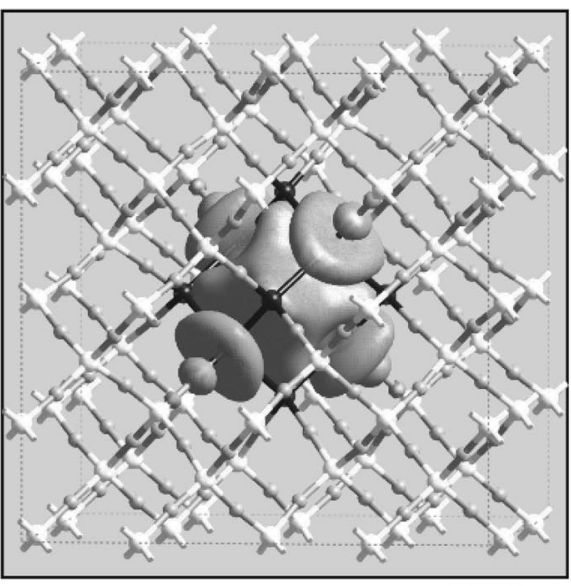

LUMO+3

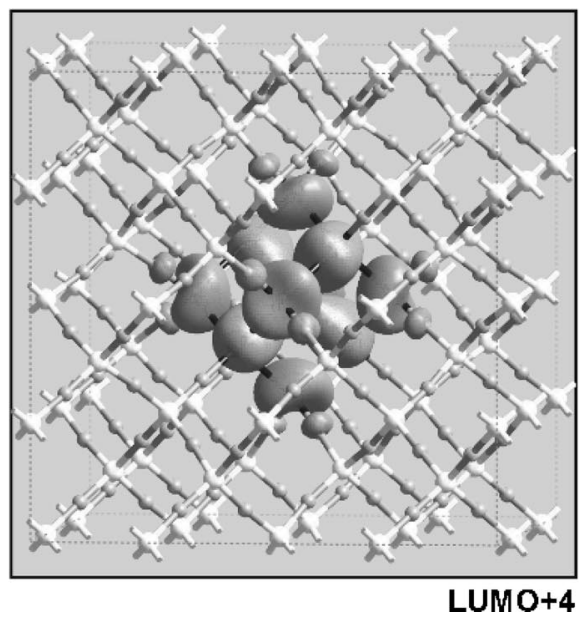

FIG. 8. Isosurfaces at fixed value ( $10 \%$ of max. amplitude) of the square modulus of the highest occupied (HOMO) and lowest but three

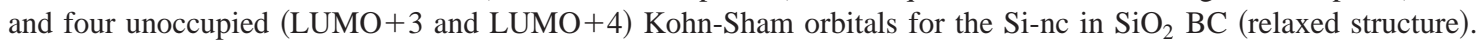

causes the formation of $\mathrm{Si}$-nc embedded into a $\mathrm{SiO}_{2}$ matrix due to a phase separation phenomenon. The core of the Si-nc is of crystalline nature as clear lattice planes are measured by EFTEM.

(2) Contrary to what happens with flat $\mathrm{Si} / \mathrm{SiO}_{2}$ interfaces, the interface between $\mathrm{Si}-\mathrm{nc}$ and $\mathrm{SiO}_{2}$ extends on a transition region.

(3) The structure and chemical composition of the region between $\mathrm{Si}$-nc and $\mathrm{SiO}_{2}$ are progressively changing from the crystalline core to amorphous silicon (as inferred from EFTEM measurements), to stressed $\mathrm{SiO}_{2}$ (as deduced from TEY-PLY comparison) and, finally, to $\mathrm{SiO}_{2}$ embedding matrix.

(4) The transition region plays an active role in the luminescence mechanism. In fact PLY data show that a strong photoluminescence signal is measured when the $\mathrm{x}$-ray energy is resonant with the stressed $\mathrm{SiO}_{2}$ absorption edge.

(5) It is not clear at this stage of the investigation whether the transition region is housing those defects which have been assumed to be active in the emission properties of Si-nc by many authors. ${ }^{45}$

(6) The passivation properties of the Si-nc surface is crucial to the understanding of their electronic structures. Indeed calculations have shown different energy band structures for hydrogenated Si-nc, isolated oxygen passivated $\mathrm{Si}-\mathrm{nc}$ and, as reported here, $\mathrm{Si}$-nc embedded into a $\mathrm{SiO}_{2}$ matrix.

(7) Total energy minimization of Si-nc shows that a significant relaxation is occurring not only in the Si-nc itself but also in the first atomic shell of $\mathrm{SiO}_{2}$ surrounding the Si-nc. Here again the presence of a transition region between Si-nc and bulk $\mathrm{SiO}_{2}$ is evidenced.

(8) Core-level energy calculations show that a stressed $\mathrm{SiO}_{2}$ may have a similar core-level shift as that observed in comparing TEY and PLY, which supports the attribution of the white peak in the PLY spectra to stressed silica.

(9) Wave function localization of some near-band-edge states on the interface Si-O bonds has been found. This implies that the optical properties of the Si-nc are determined not only by the core of the Si-nc but also by the interface region between the $\mathrm{Si}-\mathrm{nc}$ and the $\mathrm{SiO}_{2}$ where a stressed silica is expected.

What clearly appears from all these points is the fact that the properties of $\mathrm{Si}$-nc in $\mathrm{SiO}_{2}$ are fundamentally different from those of $\mathrm{Si}$-nc in porous silicon. It seems not correct to use porous silicon as representative of silicon nanocrystals. Indeed both the optical and the emission properties of the two materials may differ because of the active role played by the transition region between $\mathrm{Si}$ and $\mathrm{SiO}_{2}$.

\section{CONCLUSION}

In conclusion, we have performed an experimental and theoretical study of the properties of light emitting Si-nc embedded in a silica matrix produced by PECVD and thermal annealing. Our results indicate the presence of a transition region between the $\mathrm{Si}-\mathrm{nc}$ and the $\mathrm{SiO}_{2}$ matrix, which participates in the optical process. These experimental and theoretical analyses clearly point out the important role played not only by the Si-nc but also by a modified silica capping region in determining the optoelectronic properties of this system. Its relevance for the emission properties of Si-nc and for the observed optical gain in Si-nc is under investigation.

\section{ACKNOWLEDGMENTS}

This work was supported by INFM, under the advanced research project PRA-RAMSES and Large Facilities grants. All the calculations were performed at the CINECA (Bologna-Italy) and CICAIA (Modena-Italy) supercomputing facilities. We acknowledge the support by the Training and Mobility of Researchers (TMR) Program of the European Community for measurements at the LURE Laboratories (Orsay-France), and by MIUR through the project FIRB. 
*E-mail address: luppim@unimo.it, luppi@few.vu.nl

${ }^{1}$ A. A. Seraphin, S. T. Ngian, and K. D. Kolenbrander, J. Appl. Phys. 80, 6429 (1996).

${ }^{2}$ T. van Buuren, L. N. Dinh, L. L. Chase, W. J. Siekhaus, and L. J. Terminello, Phys. Rev. Lett. 80, 3803 (1998).

${ }^{3}$ L. N. Dihn, W. McLean II, M. A. Schildbach, and M. Balooch, Phys. Rev. B 59, 15513 (1999).

${ }^{4}$ M. V. Wolkin, J. Jorne, P. M. Fauchet, G. Allan, and C. Delerue, Phys. Rev. Lett. 82, 197 (1999).

${ }^{5}$ H. Kageshima and K. Shiraishi, Phys. Rev. Lett. 81, 5936 (1998).

${ }^{6}$ B. Garrido, M. Lopez, O. Gonzalez, A. Perez-Rodriguez, J.R. Morante, and C. Bonafos, Appl. Phys. Lett. 77, 3143 (2000).

${ }^{7}$ L. Patrone, D. Nelson, V. I. Safarov, M. Sentis, W. Marine, and S. Giorgio, J. Appl. Phys. 87, 3829 (2000).

${ }^{8}$ E. Degoli and S. Ossicini, Surf. Sci. 470, 32 (2000).

${ }^{9}$ A. Puzder, A. J. Williamson, J. C. Grossman, and G. Galli, Phys. Rev. Lett. 88, 097401 (2002).

${ }^{10}$ I. Vasiliev, J.R. Chelikowsky, and R.M. Martin, Phys. Rev. B 65, 121302(R) (2002).

${ }^{11}$ A. B. Filonov, S. Ossicini, F. Bassani, and F. Arnaud d'Avitaya, Phys. Rev. B 65, 195317 (2002).

${ }^{12}$ M. Luppi and S. Ossicini, J. Appl. Phys. 94, 2130 (2003).

${ }^{13}$ L. Pavesi, L. Dal Negro, C. Mazzoleni, G. Franzò, and F. Priolo, Nature (London) 408, 440 (2000).

${ }^{14}$ L. Dal Negro, M. Cazzanelli, N. Daldosso, Z. Gaburro, L. Pavesi, F. Priolo, D. Pacifici, G. Franzò, and F. Iacona, Physica E 16, 297 (2003).

${ }^{15}$ F. Iacona, G. Franzò, and C. Spinella, J. Appl. Phys. 87, 1295 (2000)

${ }^{16}$ G. Vijaya Prakash, N. Daldosso, E. Degoli, F. Iacona, M. Cazzanelli, Z. Gaburro, G. Puker, P. Dalba, F. Rocca, G. Franzò, E. Ceretta Moreira, D. Pacifici, F. Priolo, C. Arcangeli, A. B. Filonov, S. Ossicini, and L. Pavesi, J. Nanosci. Nanotechnol. 1, 159 (2001).

${ }^{17}$ A. Erbil, G. S. Cargill III, R. Frahm, and R. F. Boehme, Phys. Rev. B 37, 2450 (1988).

${ }^{18}$ For a general introduction to the problem, see J. Sthör, NEXAFS Spectroscopy (Springer-Verlag, Berlin, 1992).

${ }^{19}$ See also T. K. Sham, S. J. Naftel and I. Coultard, in Chemical Applications of Synchrotron Radiation, Part II X-ray Applications, Vol. 12b of Advanced Series in Physical Chemistry edited by T. K. Sham (World Scientific, Singapore 2002); Chap. 25, pp. 1154-1212.

${ }^{20}$ M. Kasrai, W. N. Lennard, R. W. Brunner, G. M. Bancroft, J. A. Bardwell, and K. T. Tan, Appl. Surf. Sci. 99, 303 (1996).

${ }^{21}$ For a general review of PLY-XAS, see A. Rogalev and J. Goulon, in Chemical Applications of Synchrotron Radiation, PartII X-ray Applications (Ref. 19) Chap. 15, pp. 707-760.

${ }^{22}$ N. Daldosso, Ph.D. thesis, Université J. Fourier, Grenoble, France 2001.
${ }^{23}$ F. Rocca, G. Dalba, N. Daldosso, Asian J. Phys. 9, 759 (2000).

${ }^{24}$ N. Daldosso, G. Dalba, P. Fornasini, R. Grisenti, and F. Rocca, Phys. Status Solidi A 197, 99 (2003).

${ }^{25}$ G. Dalba, N. Daldosso, P. Fornasini, M. Grimaldi, R. Grisenti, and F. Rocca, Phys. Rev. B 62, 9911 (2000).

${ }^{26}$ G. Dalba, N. Daldosso, P. Fornasini, R. Graziola, R. Grisenti, and F. Rocca, J. Non-Cryst. Solids 232, 370 (1998).

${ }^{27}$ G. Dalba, P. Fornasini, R. Grisenti, N. Daldosso, and F. Rocca, Appl. Phys. Lett. 74, 1454 (1999).

${ }^{28}$ G. Dalba, N. Daldosso, P. Fornasini, R. Grisenti, L. Pavesi, F. Rocca, G. Franzò, F. Priolo, and F. Iacona, Appl. Phys. Lett. 82, 889 (2003).

${ }^{29}$ P. Lagarde, A.M. Flank, and J.P. Itiè, Jpn. J. Appl. Phys. 32, 613 (1993).

${ }^{30}$ G. Dalba, N. Daldosso, D. Diop, P. Fornasini, R. Grisenti, and F. Rocca, J. Lumin. 80, 103 (1999).

${ }^{31}$ J. Chaboy, M. Benfatto, and I. Davoli, Phys. Rev. B 52, 10014 (1995).

${ }^{32}$ C. Levelut, D. Cabaret, M. Benoit, P. Jund, and A.-M. Flank, J. Non-Cryst. Solids 293-295, 100 (2001).

${ }^{33}$ W.H. McMaster, N. Kerr Del Grande, and J.H. Hubbell, Compilation of X-ray Cross-Sections (National Technical Information Service, Springfield, VA, 1969).

${ }^{34}$ A. Pasquarello, M.S. Hybertsen, and R. Car, Appl. Surf. Sci. 104/ 105, 317 (1996).

${ }^{35}$ H. Kageshima, K. Shiraishi, Proceedings of the 23rd International Conference on the Physics of Semiconductors, edited by M. Scheffler and R. Zimmermann (World Scientific, Singapore, 1996), p. 903.

${ }^{36}$ H. Kageshima and K. Shiraishi, Phys. Rev. Lett. 81, 5936 (1998).

${ }^{37}$ A. Thean and J.P. Leburton, J. Appl. Phys. 90, 6384 (2001).

38 46. H.-Ch. Weissker, J. Furthmüller, and F. Bechstedt, Phys. Rev. B 65, 155327 (2002).

${ }^{39}$ R. Sammynaiken, S.J. Naftel, T.K. Sham, K.W. Cheah, B. Averboukh, R. Huber, Y.R. Shen, G.G. Qin, Z.C. Ma, and W.H. Zong, J. Appl. Phys. 92, 3000 (2002).

${ }^{40}$ N. Ikarashi and K. Watanabe, Jpn. J. Appl. Phys. 39, 1278 (2000).

${ }^{41}$ CAMBRIDGE SERIAL TOTAL ENERGY PACKAGE, Version 4.2.1, 2000; V. Milman, B. Winkler, J.A. White, C.J. Pickard, M.C. Payne, E.V. Akhmatskaya, and R.H. Nobes, Int. J. Quantum Chem. 77, 895 (2000).

${ }^{42}$ CERIUS $^{2}$ User Guide and MATERIALS STUDIO 2.1.5 Users Guide for CASTEP, MSI, Accelrys Inc. 2000-2002.

${ }^{43}$ D. Vanderbilt, Phys. Rev. B 41, 7892 (1990).

${ }^{44}$ J.P. Perdew, K. Burke, and M. Ernzerhof, Phys. Rev. Lett. 80, 891 (1998).

${ }^{45}$ L. Khriachtchev, S. Novikov, and J. Lahtinen J. Appl. Phys. 92, 5856 (2002). 